June 2002

hep-th/0206187

US-02-05

\title{
Fermions in Kaluza-Klein and Randall-Sundrum Theories ${ }^{1}$
}

\author{
Shoichi ICHINOSE ${ }^{2}$
}

Laboratory of Physics, School of Food and Nutritional Sciences, University of Shizuoka, Yada 52-1, Shizuoka 422-8526, Japan

\begin{abstract}
The Kaluza-Klein theory and Randall-Sundrum theory are examined comparatively, with focus on the behavior of the five dimensional (Dirac) fermion in the dimensional reduction to four dimensions. They are properly treated using the Cartan formalism. In the KK case, the dual property between the electric and magnetic dipole moments is revealed in relation to the ratio of two massive parameters: the inverse of the radius of the extra-space circle and the 5D fermion mass. The order estimation of the couplings is done. In the RS case, we consider the interaction with the 5D(bulk) Higgs field and the gauge field. The chiral property, localization, anomaly phenomena are examined. We evaluate the bulk quantum effect using the method of the induced effective action. The electric dipole moment term naturally appears. This is a new origin of the CP-violation. In the $4 \mathrm{D}$ limit, the dual relation between KK model and RS model appears.

\footnotetext{
${ }^{1}$ This work is based on the content presented at the Fifth KEK Topical Conference (2001.11.19-22, Tsukuba, Japan) [1]

${ }^{2}$ E-mail address: ichinose@u-shizuoka-ken.ac.jp
} 
PACS NO: 04.50.+h, 11.10.Kk, 11.25.Mj, 12.10.-g 11.30.Er, 13.40.Em, Key Words: Kaluza-Klein theory, Randall-Sundrum theory, Cartan formalism, Extra dimension, Massless chiral fermion, Localization, Electric and magnetic dipole moment, $\mathrm{CP}$-violation

\section{Introduction}

If the present research direction of the unification of forces, using strings and D-branes, is right, the unified theory should be, effectively at some scale, some higher-dimensional field theory. Then the real world of 4 dimensions could be viewed as some "approximation" of the higher dimensional one. This procedure is called dimensional reduction. There are two representative and contrastive approaches: Kaluza-Klein (KK) and Randall-Sundrum (RS) theories. At present there seems to be no strong reason to believe that one is better than the other. Both have advantages and disadvantages. From the viewpoint of the space-time unification of gauge theories, KK reduction looks attractive. The gauge fields are nicely realized from the space-time symmetry. On the other hand, RS reduction looks attractive in some physicallyinteresting points such as the chirality control, the massless localized fermion and the anomaly flow. In fact many literatures on both theories have been produced and are now being produced. In the present paper we compare the two approaches and reveal their important features by contrasting them.

The Kaluza-Klein theory has the long history.[2, 3] It is characterized by compactifying the extra manifold. In this procedure the radius of the compact manifold, $1 / \mu$, is introduced as the size parameter. On the other hand, in the Randall-Sundrum model $[4,5]$, the localized configuration in the extra space is utilized, instead of compactifying the extra space. In this procedure the size parameter, $1 / k$, of the localization ("thickness" of the wall) is introduced. Both approaches accomplish the dimensional reduction by adjusting the size parameters.

In the original papers by Randall and Sundrum, the comparison between the two models is made mainly in the mass hierarchy. Here we focus on another aspect, that is, the magnetic and electric dipole moment terms. They are both higher-dimensional operators (the mass-dimension 5). The former one usually appears, in the $4 \mathrm{D} \mathrm{QED}$, as a quantum effect. The latter one is a $C P$-violation term. Both ones are very important in phenomenology 
and experiments. (Their measurement could become a strong candidate to confirm a higher dimensional unification model. Estimation of the dipole moments for various elementary particles, from the naturalness requirement, has been done in a recent interesting paper [6].) The appearance of the CPviolating term in the KK model was presented by Thirring very long ago[7]. The results are still important and interesting even at the present time, but the work was done so many years ago that the content is not familiar to most readers. Therefore we explain it, in the present viewpoint, in Sec.2 and 3. We regard it as a proto-type to investigate the dimensional reduction and use it for the comparison with the RS case. We analyze the higher-dimensional terms in the RS model. The estimated magnitude of the electric dipole moment (EDM) and the magnetic dipole moment (MDM) in KK model is too small to observe, while that in the RS model can be expected to escape the case. As other approaches of CP-violation, in the brane world context, we refer to ref.[8, 9].

In order to properly treat fermions in the general relativity, we take the Cartan formalism[10] (See a textbook[11, 12, 13]. ). It clarifies the gauge structure of the system on the local Lorentz frame. We explain it both for the KK and for the RS geometries. Recently many brane world models are discussed, where the fermion part is important in relation to the chiral property. Because the chiral property much depends on the delicate points such as the signs $( \pm)$ or the phases $( \pm i)$, it is worthwhile presenting a strict formulation of the fermion theory at the present stage of the development. In the analysis, we take a stable brane world model[5, 14], that is one wall RS model.

The paper consists of the following contents. In Sec.2 we review the KK theory in the Cartan formalism. It is compared with the case of the RS theory in Sec.4 and App.B. Sec.3 treats the Dirac fermion in KK theory, where the electric and magnetic dipole moments appear and the dual property is stressed. In Sec.4, Randall-Sundrum theory is explained in the Cartan formalism. The Dirac fermion in the RS theory is explained in Sec.5. The RS counterpart of the KK expansion appears. The eigen functions are characterized by the Bessel differential equation. The bulk (5D) Higgs mechanism is explained in Sec.6, where the localization of the fermion is explained. The fermion gets a mass through the Yukawa interaction with the bulk Higgs. The 5D QED is examined in Sec.7, where the 5D bulk quantum effects produce the anomaly flow and the electric dipole moment in the effective action. 
We conclude in Sec.8. Two appendixes are provided for clarifying the text.

\section{Kaluza-Klein Theory}

Let us first review the 5D Kaluza-Klein theory. This serves as the preparation for a similar treatment of the Randall-Sundrum theory. The 5D space-time manifold is described by the 4D coordinates $x^{a}(a=0,1,2,3)$ and an extra coordinate $y$. We also use the notation $\left(X^{m}\right)=\left(x^{a}, y\right),(m=0,1,2,3,5)$. With the general 5D metric $\hat{g}_{m n}$,

$$
d s^{2}=\hat{g}_{m n}(X) d X^{m} d X^{n},
$$

we assume the $S^{1}$ compactification condition for the extra space.

$$
\hat{g}_{m n}(x, y)=\hat{g}_{m n}\left(x, y+\frac{2 \pi}{\mu}\right),
$$

where $\mu^{-1}$ is the radius of the extra space circle. We specify the form of the metric as

$$
d s^{2}=g_{a b}(x) d x^{a} d x^{b}+\mathrm{e}^{2 \sigma(x)}\left(d y-f A_{a}(x) d x^{a}\right)^{2},
$$

where $g_{a b}(x), A_{a}(x)$ and $\sigma(x)$ are all $4 \mathrm{D}$ quantities, namely, the 4D metric, the $\mathrm{U}(1)$ gauge field and the dilaton (Weyl scale) field, respectively. $f$ is a coupling constant. This specification is based on the following additional assumptions.

1. $y$ is a space coordinate.

2. The geometry is invariant under the $\mathrm{U}(1)$ symmetry: $y \rightarrow y+\Lambda(x), A_{a}(x) \rightarrow$

$$
A_{a}(x)+\frac{1}{f} \partial_{a} \Lambda \text {. }
$$

The $S^{1}$ extra space $\{y\}$ is here identified as the "U(1) gauge parameter space". (This way of unification of the gauge theory and gravity should be compared with the treatment in the RS approach where the gauge parameter space is introduced as an internal one. )

We can generalize (3), keeping the periodicity condition (2), by taking the following ones instead of $g_{a b}(x), A_{a}(x)$ and $\sigma(x)$ of (3),

$$
g_{a b}(X)=\sum_{k \in \mathbf{Z}} \tilde{g}_{a b}^{(k)}(x) \mathrm{e}^{i k \mu y}, A_{a}(X)=\sum_{k \in \mathbf{Z}} \tilde{A}_{a}^{(k)}(x) \mathrm{e}^{i k \mu y}, \sigma(X)=\sum_{k \in \mathbf{Z}} \tilde{\sigma}^{(k)}(x) \mathrm{e}^{i k \mu y},
$$


where $\mathbf{Z}$ is the set of all integers. The appearance of the periodic function $\left\{\mathrm{e}^{i k \mu y} \mid k \in \mathbf{Z}\right\}$ is a character of the KK approach. The eq.(3) is the zero-th ( $k=0$, massless) mode of (4). We do not examine, in this paper, the role of the massive modes $(\mathrm{k} \neq 0)$ for these boson fields.

We take the Cartan formalism to introduce Dirac fermions and to compute the geometric quantities such as the connection and the Riemann curvature[7]. The basis $\left\{\hat{\theta}^{\mu}\right\}$ of the cotangent manifold $\left(T_{p}^{*} M\right)$ can be defined as the 1-form which satisfies the relation:

$$
d s^{2}=\hat{\theta}^{\mu} \hat{\theta}^{\nu} \hat{\eta}_{\mu \nu} \quad, \quad \operatorname{diag}\left(\hat{\eta}_{\mu \nu}\right)=(-1,1,1,1,1),
$$

where $\mu, \nu=0,1,2,3, \overline{5}$ are the local Lorentz (tangent frame) indices. $d s^{2}$ is given in (3). The fünf-bein $\hat{e}_{m}^{\mu}$ is generally defined as

$$
\hat{\theta}^{\mu}=\hat{e}_{m}^{\mu} d X^{m} .
$$

$\left\{\hat{\theta}^{\mu}\right\}$ is the dual of the basis $\left\{\hat{e}^{\mu} \equiv \hat{e}^{\mu m} \partial / \partial X^{m}\right\}$ of the tangent manifold $\left(T_{p} M\right) . \quad\left(<\hat{e}^{\mu}, \hat{\theta}_{\nu}>=\delta_{\nu}^{\mu}.\right)$ For the $4 \mathrm{D}$ part, we denote as $\hat{\theta}^{\alpha}=\theta^{\alpha}(\alpha=$ $0,1,2,3)$ which satisfies

$$
\begin{array}{r}
g_{a b}(x) d x^{a} d x^{b}=e^{\alpha}{ }_{a}(x) e^{\beta}{ }_{b}(x) \eta_{\alpha \beta} d x^{a} d x^{b}=\theta^{\alpha} \theta^{\beta} \eta_{\alpha \beta}, \\
\theta^{\alpha}=e_{a}^{\alpha}(x) d x^{a},
\end{array},
$$

where $g_{a b}$ is the same as (3), $e_{a}^{\alpha}$ and $\eta_{\alpha \beta}$ are the $4 \mathrm{D}$ part of the fünf-bein $\hat{e}^{\mu}{ }_{m}$ and the 5D flat (Minkowski) metric $\hat{\eta}_{\mu \nu}$ respectively. $\left(e^{\alpha}{ }_{a}=\hat{e}_{a}^{\alpha}, \eta_{\alpha \beta}=\hat{\eta}_{\alpha \beta}\right)^{3}$ The extra component $\hat{\theta}^{\overline{5}}$ can be read off, from (3), as

$$
\hat{\theta}^{\overline{5}}=e^{\sigma}\left(d y-f A_{a} d x^{a}\right) .
$$

Hence the fünf-bein $\hat{e}_{m}^{\mu}$ and its inverse $\hat{e}_{\mu}^{m}$ can be concretely given by

$$
\begin{gathered}
\left(\hat{e}_{m}^{\mu}{ }_{m}\right)=\left(\begin{array}{cc}
e^{\alpha}{ }_{a} & 0 \\
-f e^{\sigma} A_{a} & e^{\sigma}
\end{array}\right),\left(\hat{e}_{\mu}{ }^{m}\right)=\left(\begin{array}{cc}
e_{\alpha}^{a} & 0 \\
f A_{\alpha} & e^{-\sigma}
\end{array}\right), \\
\hat{e}_{m}^{\mu} \hat{e}_{\mu}^{n}=\delta_{m}^{n} \quad, \quad \hat{e}_{m}^{\mu}{ }_{m} \hat{e}_{\nu}{ }^{m}=\delta_{\nu}^{\mu} \quad, \quad A_{\alpha} \equiv e_{\alpha}^{a} A_{a} .
\end{gathered}
$$

\footnotetext{
${ }^{3}$ The suffix notation taken in this article is as follows: $m, n, \cdots$ (Roman alphabets beginning from the middle) are for $1+4 \mathrm{D}$ general space-time coordinates; $a, b, \cdots$ (Roman alphabets beginning from the first) are for $1+3 \mathrm{D}$ general space-time coordinates; $\mu, \nu, \cdots$ (Greek symbols beginning from the middle) are for $1+4 \mathrm{D}$ local Lorentz (Minkowski) coordinates; $\alpha, \beta, \cdots$ (Greek symbols beginning from the first) are for $1+3 \mathrm{D}$ local Lorentz (Minkowski) coordinates. The 5-th component is explicitly denoted as " 5 " for the general coordinate and as $\overline{5}$ for the local Lorentz one. The hat symbol " " " is used to discriminate $5 \mathrm{D}$ symbols from $4 \mathrm{D}$ symbols when it is necessary.
} 
The first Cartan's structure equation, for the torsionless case $\left(\hat{T}^{\mu}=0\right)$, is given by

$$
d \hat{\theta}^{\mu}+\hat{\omega}_{\nu}^{\mu} \wedge \hat{\theta}^{\nu}=\hat{T}^{\mu}=0 \quad, \quad \hat{\omega}_{\mu \nu}+\hat{\omega}_{\nu \mu}=0
$$

where $\hat{\omega}^{\mu}{ }_{\nu}$ is the connection 1-form. From this equation and eqs.(7) and (8), we can read the connection as

$$
\begin{aligned}
& \hat{\omega}_{\overline{5}}^{\overline{5}_{\overline{5}}}=-\frac{1}{2} \partial_{a} \sigma d x^{a} \quad, \quad \hat{\omega}_{\alpha}^{\overline{5}}=-\hat{\omega}_{\alpha}^{\overline{5}}=\frac{1}{2} \partial_{a} \sigma e_{\alpha}^{a} \hat{\theta}^{\overline{5}}-\frac{f}{2} e^{\sigma} F_{\alpha \beta} \hat{\theta}^{\beta}, \\
& \hat{\omega}_{\beta}^{\alpha}=\omega_{\beta}^{\alpha}+\frac{f}{2} e^{\sigma} F_{\beta}^{\alpha} \hat{\theta}^{\overline{5}}, \\
& F_{a b}=\partial_{a} A_{b}-\partial_{b} A_{a} \quad, \quad F_{\alpha \beta} \equiv e_{\alpha}{ }^{a} e_{\beta}^{b} F_{a b} \quad,
\end{aligned}
$$

where $\omega_{\beta}^{\alpha}$ is the $4 \mathrm{D}$ connection which is defined by $d \theta^{\alpha}+\omega_{\beta}^{\alpha} \wedge \theta^{\beta}=0$. (Note that $\omega_{\beta}^{\alpha} \neq \hat{\omega}_{\beta}^{\alpha}$.)

The second Cartan's structure equation is given by

$$
d \hat{\omega}_{\mu \nu}+\hat{\omega}_{\mu \sigma} \wedge \hat{\omega}_{\nu}^{\sigma}=\frac{1}{2} \hat{R}_{\mu \nu \sigma \tau} \hat{\theta}^{\sigma} \wedge \hat{\theta}^{\tau}
$$

This gives the curvatures $\hat{R}_{\mu \nu \sigma \tau}$ as

$$
\begin{gathered}
\hat{R}_{\alpha \beta \gamma \delta}=R_{\alpha \beta \gamma \delta}-\frac{f^{2}}{2} e^{2 \sigma} F_{\alpha \beta} F_{\gamma \delta}+\frac{f^{2}}{4} e^{2 \sigma}\left(F_{\alpha \delta} F_{\beta \gamma}-\delta \leftrightarrow \gamma\right), \\
\hat{R}_{\alpha \overline{5} \overline{5} \beta}=\frac{f^{2}}{4} e^{2 \sigma} F_{\alpha \gamma} F_{\beta}^{\gamma}+\cdots
\end{gathered}
$$

Hence the 5D Riemann scalar curvature $\hat{R}_{\nu}^{\mu}{ }_{\mu}$ can be decomposed as

$$
\hat{R}=R+\frac{f^{2}}{4} e^{2 \sigma} F^{\alpha \beta} F_{\alpha \beta}+\frac{1}{2} \partial_{a} \sigma \partial_{b} \sigma g^{a b}+\frac{1}{2} \nabla^{2} \sigma
$$

which shows the theory of gravity, electro-magnetism and the dilaton in the $4 \mathrm{D}$ world. The correct sign appears in front of $F^{\alpha \beta} F_{\alpha \beta}$, which comes from the choice of the extra coordinate $y$, in (3), as a space (not time) component. This point was stressed by Thirring[7].

We consider the simple case $\sigma=0$ for the present purpose. 


\section{Fermions in Kaluza-Klein Theory}

The 5D Dirac equation is generally given by (see a textbook $[11,12,13]$ )

$$
\left\{\gamma^{\mu} \hat{e}_{\mu}^{m} \frac{\partial}{\partial X^{m}}+\frac{1}{8}\left(\hat{\omega}^{\sigma}\right)_{\mu \nu} \gamma_{\sigma}\left[\gamma^{\mu}, \gamma^{\nu}\right]+\hat{m}\right\} \hat{\psi}=0
$$

$\hat{m}$ is the mass parameter of the $5 \mathrm{D}$ fermion $(-\infty<\hat{m}<\infty)$. ${ }^{4}$ The spin connection above $\left(\hat{\omega}^{\sigma}\right)_{\mu \nu}$ is defined by $\hat{\omega}^{\mu}{ }_{\nu}=\left(\hat{\omega}_{\lambda}\right)^{\mu}{ }_{\nu} \hat{\theta}^{\lambda}$. (Local Lorentz suffixes are lowered or raised by the 5D Minkowski metric $\hat{\eta}_{\mu \nu}$ or $\hat{\eta}^{\mu \nu}$ respectively.) The 5D Dirac matrix $\gamma^{\mu}$ satisfies $\left\{\gamma^{\mu}, \gamma^{\nu}\right\}=2 \hat{\eta}^{\mu \nu}$. For simplicity we switch off the 4D gravity: $e_{a}^{\alpha} \rightarrow \delta_{a}^{\alpha}, \omega_{\beta}^{\alpha} \rightarrow 0$. In the present case, using (9) and (11), the eq.(15) says

$$
\left\{\gamma^{a}\left(\partial_{a}+f A_{a} \partial_{5}\right)+\gamma^{5} \partial_{5}-\frac{f}{16} F_{a b} \gamma^{5}\left[\gamma^{a}, \gamma^{b}\right]+\hat{m}\right\} \hat{\psi}=0
$$

where $\partial_{5}=\partial / \partial y$.

(A) Charged Fermion

Corresponding to a massive mode in the $4 D$ reduction (4), we consider the following form for a charged fermion.

$$
\hat{\psi}(x, y)=\mathrm{e}^{i\left(\phi \gamma^{5}+\mu y\right)} \psi(x)
$$

Here we regard the charged fermion as a KK-massive mode. The angle parameter $\phi$ is chosen as

$$
\begin{array}{r}
\left(i \gamma^{5} \mu+\hat{m}\right) \mathrm{e}^{2 i \phi \gamma^{5}}=\sqrt{\hat{m}^{2}+\mu^{2}} \equiv M \\
(i) \hat{m} \neq 0: \tan 2 \phi=-\frac{\mu}{\hat{m}}, \\
-\frac{\pi}{2}<2 \phi \leq 0 \text { for } \hat{m}>0 ; \quad \pi \leq 2 \phi<\frac{3 \pi}{2} \text { for } \hat{m}<0 \\
\text { (ii) } \hat{m}=0: 2 \phi=-\frac{\pi}{2},
\end{array}
$$

where $\left(\gamma^{5}\right)^{2}=1, e^{i \alpha \gamma^{5}}=\cos \alpha+i \gamma^{5} \sin \alpha$, is used. See the lower half region of Fig.1.

\footnotetext{
${ }^{4}$ The 5D gamma matrices $\left\{\gamma^{\mu}\right\}$ are defined in App.A.
} 


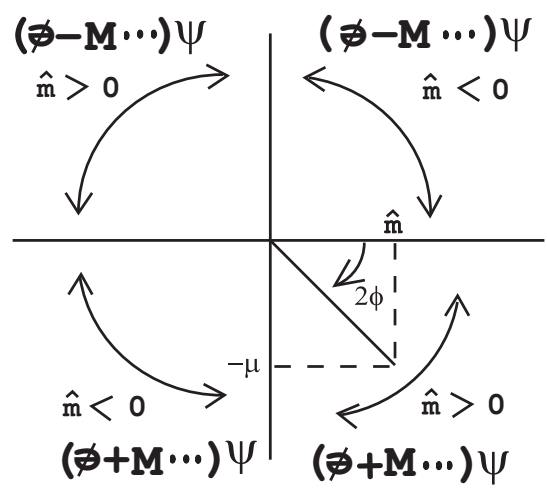

Fig. 1 The angle parameter $\phi$ which defines the 4D charged fermion in the KK dimensional reduction (17). $\hat{m}$ is the $5 \mathrm{D}$ fermion mass parameter, $\mu^{-1}$ is the size of the extra compact manifold.

$-\pi / 2<2 \phi \leq 0$ for $\hat{m}>0 ; \pi \leq 2 \phi<3 \pi / 2$ for $\hat{m}<0 ; 2 \phi=-\pi / 2$ for $\hat{m}=0$. The upper half region gives the same $4 \mathrm{D}$ fermion action as the lower half region by the transformation $\psi \rightarrow \gamma^{5} \psi$.

Then (16) reduces to

$$
\left\{\gamma^{a}\left(\partial_{a}+i e A_{a}\right)+M-\frac{1}{16 M}\left(\hat{m} \frac{e}{\mu}-i e \gamma^{5}\right) F_{a b} \gamma^{5}\left[\gamma^{a}, \gamma^{b}\right]\right\} \psi=0
$$

where $e \equiv f \mu$ is the electric coupling constant. $M$ is identified as a $4 \mathrm{D}$ fermion mass. 5 We notice, in this expression, the EDM and the MDM

${ }^{5}$ We can take the angle $\phi$ in (18) in another way.

$$
\begin{array}{r}
\left(i \gamma^{5} \mu+\hat{m}\right) \mathrm{e}^{2 i \phi \gamma^{5}}=-\sqrt{\hat{m}^{2}+\mu^{2}}=-M \\
\text { (i) } \hat{m} \neq 0: \quad \tan 2 \phi=-\frac{\mu}{\hat{m}}, \\
\frac{\pi}{2}<2 \phi \leq \pi \text { for } \hat{m}>0 ; \quad 0 \leq 2 \phi<\frac{\pi}{2} \text { for } \hat{m}<0 \\
\text { (ii) } \hat{m}=0: 2 \phi=\frac{\pi}{2},
\end{array}
$$

Then the $4 \mathrm{D}$ equation (19) is replaced by

$$
\left\{\gamma^{a}\left(\partial_{a}+i e A_{a}\right)-M+\frac{1}{16 M}\left(\hat{m} \frac{e}{\mu}-i e \gamma^{5}\right) F_{a b} \gamma^{5}\left[\gamma^{a}, \gamma^{b}\right]\right\} \psi=0
$$


naturally appear[7]. They can be written as

$$
\frac{1}{16 \mu} e F_{a b} \bar{\psi}\left(c_{E} \gamma^{5}+i c_{M}\right)\left[\gamma^{a}, \gamma^{b}\right] \psi \quad, \quad c_{E} \equiv \frac{\hat{m}}{M} \quad, \quad c_{M} \equiv \frac{-\mu}{M}
$$

The first term(EDM) violates the CP symmetry. The coefficient depends on the sign besides the absolute value of $\hat{m}$. Writing the two coefficients as $\left(c_{E}, c_{M}\right) \equiv(\hat{m} / M,-\mu / M)$, we can consider the following two limits:

(i) CP-preserved limit [Small radius limit, 4D limit ] ${ }^{6}$

$\hat{m} / \mu \rightarrow \pm 0,\left(c_{E}, c_{M}\right)=( \pm 0,-1), 2 \phi \rightarrow-(\pi / 2-0)[3 \pi / 2-0]$ for upper [lower] sign;

(ii) CP-extremely-violated limit [Large radius limit, 5D limit ] $\hat{m} / \mu \rightarrow \pm \infty,\left(c_{E}, c_{M}\right)=( \pm 1,0), 2 \phi \rightarrow-0[\pi+0]$ for upper [lower] sign.

Note that the MDM term usually appears, in the 4D QED, as the quantum effect.

(B) Neutral Fermion

We regard the neutral fermion as the zero mode of the KK-expansion.

$$
\begin{array}{r}
\hat{\psi}(x, y)=\psi(x) \\
\left\{\gamma^{a} \partial_{a}-\frac{f}{16} F_{a b} \gamma^{5}\left[\gamma^{a}, \gamma^{b}\right]+\hat{m}\right\} \psi(x)=0
\end{array}
$$

Only the EDM term appears. This case is also realized by letting $e \rightarrow 0, \mu \rightarrow$ 0 keeping $e / \mu=f$ (fixed) in (19). Although the fermion has no charge, the dipole moment appears. This case is similar to the limit (ii).

Let us do the order estimation. From the reduction

$$
S=-\frac{1}{2 G_{5}} \int d^{5} X \sqrt{-\hat{g}} \hat{R}=-\frac{1}{2 G_{5}} \frac{2 \pi}{\mu} \int d^{4} x \sqrt{-g}\left(R+\frac{f^{2}}{4} F^{\alpha \beta} F_{\alpha \beta}+\cdots\right)
$$

See the upper half region of Fig.1. But this case reduces to (19) by the transformation $\psi \rightarrow \gamma^{5} \psi$.

${ }^{6}$ If we regard the $4 \mathrm{D}$ charged fermion as the $N$-th $\mathrm{KK}$ mode, $\mu$ in (17) is replaced by $N \mu$. Then this CP-preserved limit can also be regarded as large $N$ limit. 
we know

$$
\frac{1}{G_{5} \mu} \sim \frac{1}{G} \quad, \quad \frac{f^{2}}{G_{5} \mu} \sim 1
$$

where $G$ is the (4D) gravitational constant. This gives $f \sim \sqrt{G}=10^{-19} \mathrm{GeV}^{-1}$. On the other hand, we know $e=\mu f \sim 10^{-1}$. Hence we obtain $\mu \sim 10^{-1} f^{-1} \sim$ $10^{18} \mathrm{GeV}$. We originally have four parameters, $\hat{m}, \mu, G_{5}$ and $f$. Among them we have the two relations (25) from the observation. We have another restriction among them from the present theoretical knowledge. The most natural interpretation of the parameters is that $\mu^{-1}$ is the infrared regularization, $\hat{m}$ is the energy scale of this $5 \mathrm{D}$ KK system. Then the validity of the 5D classical treatment requires that the $5 \mathrm{D}$ Planck mass $\gg|\hat{m}|$ :

$$
\frac{1}{\sqrt[3]{G_{5}}} \sim \sqrt[3]{100} \mu \gg|\hat{m}|
$$

and this reduces to, through the previous parameter relations and values, $|\hat{m}| \ll 10^{19} \mathrm{GeV}$.

Now we consider the following three cases in order to evaluate the EDM and MDM couplings.

(0) $|\hat{m}| \sim \mu$

We can estimate the electric and magnetic couplings as

$$
\frac{|\hat{m}|}{M \mu} e \sim \frac{e}{M} \sim 10^{-32} \text { e cm } .
$$

Both electric and magnetic moment terms equally appear. In this case, however, the theoretical restriction (26) is not so well satisfied.

(i) $|\hat{m}| \ll \mu$ (small radius, 4D limit), CP-preserved limit

We can estimate as

$$
\frac{\hat{m}}{M \mu} e \sim \frac{\hat{m}}{\mu} \times 10^{-32} \text { e cm }, \frac{e}{M} \sim \frac{e}{\mu} \sim 10^{-32} \text { e cm },\left|c_{E}\right| \ll\left|c_{M}\right| .
$$

In this case the EDM coupling is suppressed by the factor of the mass parameter ratio $\frac{|\hat{m}|}{\mu}(\ll 1)$. The theoretical restriction (26) is satisfied, hence this parameter region is well controlled theoretically.

(ii) $|\hat{m}| \gg \mu$ (large radius, 5D limit), CP-extremely-violated limit We can estimate as

$$
\frac{|\hat{m}|}{M \mu} e \sim \frac{e}{\mu} \sim 10^{-32} \text { e cm } \quad, \quad \frac{e}{M} \sim \frac{\mu}{|\hat{m}|} \times 10^{-32} \text { e cm , }\left|c_{E}\right| \gg\left|c_{M}\right|
$$


In this case the MDM coupling is suppressed by the factor $\frac{\mu}{|\hat{m}|}(\ll 1)$. The theoretical restriction (26), however, is not satisfied. This implies the 5D quantum effect can not be negligible in this parameter region.

We note that the ratio of the two massive parameters, (the radius of the extra space $)^{-1} \mu$ and the 5D fermion mass $\hat{m}$, controls the dual aspect (electric versus magnetic) of the theory. This point will be compared with the RS case later (see the ending paragraphs of Sec.7).

As for the EDM, all cases are far below the experimental upper bound, for example, the neutron EDM is less than $6.3 \times 10^{-26} \mathrm{e} \mathrm{cm}[15]$. As for the MDM, we know, (from the formula of the quantum effect of 4D QED: $e \hbar / 2 m c$,) the order of the observed values are $10^{-11} \mathrm{e} \mathrm{cm}$ for the electron, $10^{-14} \mathrm{e} \mathrm{cm}$ for the proton, $10^{-16} \mathrm{e} \mathrm{cm}$ for the top quark. The prediction of $5 \mathrm{D} \mathrm{KK}$ theory is superweak compared with these values. Hence the present model is viable but quantitatively not so attractive. ${ }^{7}$ We are, at present, content with the qualitatively interesting point.

Thirring[7] showed that the CP-violating term appears not because the discrete symmetries (charge conjugation, parity and time reversal) do not exist in the Dirac equation (19), but because they appear in the form which differs from the ordinary one.

\section{Randall-Sundrum Theory in the Cartan For- malism}

Let us formulate the RS theory in the Cartan formalism. We consider the following $5 \mathrm{D}$ space-time geometry $[4,5]$.

$$
\begin{array}{r}
d s^{2}=\mathrm{e}^{-2 \sigma(y)} \eta_{a b} d x^{a} d x^{b}+d y^{2}=\hat{g}_{m n} d X^{m} d X^{n}, \\
-\infty<y<+\infty,-\infty<x^{a}<+\infty
\end{array}
$$

where $\sigma(y)$ is a "scale factor" field. $\left(\eta_{a b}\right)=\operatorname{diag}(-1,1,1,1)$. When the geometry is $\mathrm{AdS}_{5}, \sigma(y)=c|y|, c>0$. [ Such a situation, in the present case,

\footnotetext{
${ }^{7}$ The 5D KK model has another phenomenological defect in the prediction of the 4D charged fermion mass. The order of the magnitude is $M=\sqrt{\hat{m}^{2}+\mu^{2}} \sim \mu \sim 10^{18} \mathrm{GeV}$, which cannot be accepted as the ordinary (charged) fermion masses. From this, the MDM value essentially does not improve even if the quantum effect (of the $4 \mathrm{D}$ QED with the Planck mass fermion) is taken into account.
} 
occurs in the asymptotic region of the extra space $y \rightarrow \pm \infty .[14,21]]$ Note that the "scale factor" field $\sigma(y)$ depends only on the extra coordinate $y$ and controls the 4D metric part $\eta_{a b} d x^{a} d x^{b}$, whereas the corresponding field $\sigma(x)$ in the KK case of (3) depends only on the $4 \mathrm{D}$ coordinates $x^{a}$ and mainly controls the extra part $(d y)^{2}$. As in Sec.2, we introduce the local Lorentz frame as

$$
d s^{2}=\hat{\theta}^{\mu} \hat{\theta}^{\nu} \hat{\eta}_{\mu \nu} \quad, \quad\left(\hat{\eta}_{\mu \nu}\right)=\operatorname{diag}(-1,1,1,1,1) \quad .
$$

The 1 -form $\hat{\theta}^{\mu}$, the basis of the cotangent manifold $T_{p}^{*} M$, is given by

$$
\hat{\theta}^{\alpha}=\mathrm{e}^{-\sigma} \eta_{a}^{\alpha} d x^{a} \quad, \quad \hat{\theta}^{5}=d y \quad
$$

from which we can read off the fünf-bein $\hat{e}_{m}^{\mu}$ and its inverse $\hat{e}_{\mu}^{m}$ as

$$
\left(\hat{e}_{m}^{\mu}\right)=\left(\begin{array}{cc}
e^{-\sigma} \eta_{a}^{\alpha} & 0 \\
0 & 1
\end{array}\right) \quad, \quad\left(\hat{e}_{\mu}^{m}\right)=\left(\begin{array}{cc}
e^{\sigma} \eta_{\alpha}^{a} & 0 \\
0 & 1
\end{array}\right)
$$

Taking 1-form $\hat{\theta}^{\mu}$ of (32), and compare the results with the 1st Cartan's structure equation (10), we obtain the connection 1 -form $\hat{\omega}_{\nu}^{\mu}$ as

$\hat{\omega}_{\overline{5}}^{\overline{5}}=0 \quad, \quad \hat{\omega}_{\overline{5}}^{\alpha}=-\hat{\omega}_{\overline{5}}{ }^{\alpha}=-\sigma^{\prime} \theta^{\alpha} \quad, \quad \hat{\omega}_{\alpha}^{\overline{5}_{\alpha}}=-\hat{\omega}_{\alpha}{ }^{\overline{5}}=\sigma^{\prime} \theta_{\alpha} \quad, \quad \hat{\omega}_{\beta}^{\alpha}=0$

where $\sigma^{\prime}=\frac{d \sigma}{d y}$.

Further explanation of the RS geometry in the Cartan formalism is done in App.B where a more general coordinate is considered.

\section{Fermions in Randall-Sundrum Theory}

The 5D Dirac Lagrangian in the RS theory is given, from (15) and the results of Sec.4, as

$$
\begin{aligned}
& \sqrt{-\hat{g}} \mathcal{L}^{\text {Dirac }}=\sqrt{-\hat{g} i \hat{\psi}}\left\{\gamma^{\mu} \hat{e}_{\mu}^{m} \partial_{m}+\frac{1}{8}\left(\hat{\omega}^{\sigma}\right)_{\mu \nu} \gamma_{\sigma}\left[\gamma^{\mu}, \gamma^{\nu}\right]+\hat{m}(y)\right\} \hat{\psi} \\
& =i \mathrm{e}^{-3 \sigma} \overline{\hat{\psi}}\left\{\gamma^{a} \partial_{a}-2 \mathrm{e}^{-\sigma}\left(\sigma^{\prime}-\frac{1}{2} \partial_{y}\right) \gamma^{5}+\hat{m}(y) e^{-\sigma}\right\} \hat{\psi} \\
& =i \mathrm{e}^{-\frac{3}{2} \sigma} \overline{\hat{\psi}}\left\{\gamma^{a} \partial_{a}-2 \mathrm{e}^{-\sigma}\left(\frac{1}{4} \sigma^{\prime}-\frac{1}{2} \partial_{y}\right) \gamma^{5}+\hat{m}(y) e^{-\sigma}\right\}\left(\mathrm{e}^{-\frac{3}{2} \sigma} \hat{\psi}\right),
\end{aligned}
$$


where $\hat{m}$ is the $5 \mathrm{D}$ fermion mass $-\infty<\hat{m}<+\infty$. For the later use, we here allow $\hat{m}$ to have the $y$-dependence $: \hat{m}=\hat{m}(y){ }^{8}$ The special case of this result, $\hat{m}=0$, coincides with an equation cited in [16].

Let us do the dimensional reduction from $5 \mathrm{D}$ to $4 \mathrm{D}[17,18,16]$. We take the following form of expansion.

$$
\begin{array}{r}
\hat{\psi}(x, y)=\sum_{k}\left(\psi_{L}^{k}(x) \xi_{k}(y)+\psi_{R}^{k}(x) \eta_{k}(y)\right), \\
\gamma^{5} \psi_{L}(x)=-\psi_{L}(x) \quad, \quad \gamma^{5} \psi_{R}(x)=+\psi_{R}(x),
\end{array}
$$

where $\left\{\xi_{k}(y), \eta_{k}(y)\right\}$ is a complete set of some eigenfunctions to be determined. This expansion corresponds to (4) and (17) in the Kaluza-Klein case. The role of the periodic eigenfunctions $\left\{\mathrm{e}^{i k \mu y}\right\}$ is here played by $\left\{\xi_{k}(y), \eta_{k}(y)\right\}$. For simplicity, we consider the "5D-parity" even case for $\hat{\psi}(x, y)$.

$$
\gamma^{5} \hat{\psi}(x,-y)=+\hat{\psi}(x, y)
$$

(The odd case is similarly examined.) This requires $\xi_{n}(y)$ to be an odd function and $\eta_{n}(y)$ to be an even function with respect to the $Z_{2}$ transformation: $y \leftrightarrow-y$.

$$
\xi_{n}(-y)=-\xi_{n}(y) \quad, \quad \eta_{n}(-y)=+\eta_{n}(y)
$$

From these we get the following important boundary conditions,

$$
\xi_{n}(0)=0 \quad \text { (Dirichlet) } \quad,\left.\quad \partial_{y} \eta_{n}\right|_{y=0}=0 \quad \text { (Neumann) } \quad
$$

when $\xi_{n}(y)$ and $\partial_{y} \eta_{n}(y)$ are continuous at $y=0$. The Lagrangian reduces to

$$
\begin{array}{r}
\sqrt{-\hat{g}} \mathcal{L}^{\text {Dirac }}= \\
\sum_{n}\left\{\sum_{m}\left(\bar{\psi}_{L}^{m} \tilde{\xi}_{m}(y)+\bar{\psi}_{R}^{m} \tilde{\eta}_{m}(y)\right) \times\right. \\
\tilde{\xi}_{n}-\mathrm{e}^{-\sigma}\left(\frac{\sigma^{\prime}}{2}-\partial_{y}\right)\left(-\psi_{L}^{n}\right) \tilde{\xi}_{n}+\gamma^{a} \partial_{a} \psi_{R}^{n} \tilde{\eta}_{n}-\mathrm{e}^{-\sigma}\left(\frac{\sigma^{\prime}}{2}-\partial_{y}\right) \psi_{R}^{n} \tilde{\eta}_{n} \\
\left.+\mathrm{e}^{-\sigma} \hat{m}(y)\left(\psi_{L}^{n} \tilde{\xi}_{n}+\psi_{R}^{n} \tilde{\eta}_{n}\right)\right\},
\end{array}
$$

where we define $\tilde{\xi}_{n} \equiv \mathrm{e}^{-\frac{3}{2} \sigma} \xi_{n}$ and $\tilde{\eta}_{n} \equiv \mathrm{e}^{-\frac{3}{2} \sigma} \eta_{n}$.

\footnotetext{
${ }^{8}$ The situation is realized by the bulk Higgs mechanism treated in Sec.6.
} 
We now take the set of eigenfunctions $\left\{\tilde{\xi}_{n}, \tilde{\eta}_{n}\right\}$ as

$\mathrm{e}^{-\sigma}\left(\frac{\sigma^{\prime}}{2}-\partial_{y}\right) \tilde{\xi}_{n}+\mathrm{e}^{-\sigma} \hat{m}(y) \tilde{\xi}_{n}=m_{n} \tilde{\eta}_{n},-\mathrm{e}^{-\sigma}\left(\frac{\sigma^{\prime}}{2}-\partial_{y}\right) \tilde{\eta}_{n}+\mathrm{e}^{-\sigma} \hat{m}(y) \tilde{\eta}_{n}=m_{n} \tilde{\xi}_{n}$

which are orthnormalized as

$$
\begin{array}{r}
\int_{-\infty}^{\infty} d y \tilde{\xi}_{n}(y) \tilde{\xi}_{m}(y)=\int_{-\infty}^{\infty} d y \tilde{\eta}_{n}(y) \tilde{\eta}_{m}(y)=\delta_{n m} \\
\int_{-\infty}^{\infty} d y \tilde{\xi}_{n}(y) \tilde{\eta}_{m}(y)=0
\end{array}
$$

Then the 5D action (35) finally reduces to the sum of 4D free fermions.

$$
\int d^{5} X \sqrt{-\hat{g}} \mathcal{L}^{\text {Dirac }}=i \int d^{4} x \sum_{n}\left\{\bar{\psi}_{L}^{n}\left(\gamma^{a} \partial_{a} \psi_{L}^{n}+m_{n} \psi_{R}^{n}\right)+\bar{\psi}_{R}^{n}\left(\gamma^{a} \partial_{a} \psi_{R}^{n}+m_{n} \psi_{L}^{n}\right)\right\}
$$

The information of this fermion dynamics is now in the set of the eigen values $\left\{m_{n}\right\}$ determined by $(41)$.

From the coupled equation (41) with respect to $\tilde{\xi}_{n}$ and $\tilde{\eta}_{n}$, we get the differential equation for $\tilde{\xi}_{n}$ as

$$
\mathrm{e}^{-2 \sigma}\left[\frac{\sigma^{\prime \prime}}{2}-\frac{3}{4}{\sigma^{\prime}}^{2}+2 \sigma^{\prime} \partial_{y}-\hat{m}(y) \sigma^{\prime}+\hat{m}(y)^{2}-\partial_{y}{ }^{2}+\hat{m}(y)^{\prime}\right] \tilde{\xi}_{n}=m_{n}{ }^{2} \tilde{\xi}_{n}
$$

(We can obtain a similar one for $\tilde{\eta}_{n}$.) For simplicity, we consider the thin wall limit:

$$
\begin{array}{r}
\sigma(y)=\omega|y| \quad, \quad \sigma^{\prime}(y)=\omega \epsilon(y) \quad, \quad \sigma^{\prime \prime}(y)=2 \omega \delta(y), \\
\hat{m}(y)=\tilde{m} \epsilon(y) \quad, \quad \hat{m}^{\prime}(y)=2 \tilde{m} \delta(y),
\end{array}
$$

where $\omega(>0)$ and $\tilde{m}(>0)$ are some constants. $\epsilon(y)$ is the sign function: $\epsilon(y)=1$ for $y>0$ and $\epsilon(y)=-1$ for $y<0 \quad\left(\epsilon^{\prime}(y)=2 \delta(y)\right)$. In this limit, the equation (44) can be explicitly solved.

$\mathrm{e}^{-2 \omega|y|}\left[(\omega+2 \tilde{m}) \delta(y)-\frac{3}{4} \omega^{2}+2 \omega \epsilon(y) \partial_{y}-\tilde{m} \omega+\tilde{m}^{2}-\partial_{y}^{2}\right] \tilde{\xi}_{n}=m_{n}{ }^{2} \tilde{\xi}_{n}$,

where $\epsilon(y)^{2}=1$ is used. The presence of $\delta(y)$ indicates a singularity of the solution at $y=0$. Let us see the solution in the region $y>0$. In terms 
of a new coordinate $z \equiv \frac{1}{\omega} \mathrm{e}^{\omega y}$, the above equation reduces to the Bessel differential equation.

$$
\begin{array}{r}
\left\{\partial_{z}{ }^{2}-\frac{1}{z} \partial_{z}+\frac{1-\nu^{2}}{z^{2}}+m_{n}{ }^{2}\right\} \tilde{\xi}_{n}=0, \\
\nu=\left|\frac{\tilde{m}}{\omega}-\frac{1}{2}\right| .
\end{array}
$$

The solution $\tilde{\xi}_{n}$ is obtained as

$$
\begin{array}{r}
\tilde{\xi}_{n}(y)=\frac{1}{(\omega z)^{3 / 2}} \xi_{n}(z)=z\left\{J_{\nu}\left(m_{n} z\right)+c_{n} N_{\nu}\left(m_{n} z\right)\right\}, \\
c_{n}=-\frac{J_{\nu}\left(m_{n} / \omega\right)}{N_{\nu}\left(m_{n} / \omega\right)},
\end{array}
$$

where $c_{n}$ is determined by the Dirichlet boundary condition (39). $J_{\nu}(z)$ and $N_{\nu}(z)$ are two independent Bessel functions. With the above explicit solution of $\tilde{\xi}_{n}$, we can obtain $\tilde{\eta}_{n}$ using the first equation of (41). Another boundary condition (Neumann) on $\eta_{n}(39)$ gives us the set $\left\{m_{n}\right\}$ as the zeros of some combination of Bessel functions. ( For the special cases $\tilde{m}=0$ (no 5D fermion mass) or $\tilde{m} / \omega=1$, the eigen functions reduce to $J_{1 / 2}(z)=$ $\sqrt{2 / \pi z} \sin z, N_{1 / 2}(z)=-J_{-1 / 2}(z)=-\sqrt{2 / \pi z} \cos z$. The former case is that one considered in [16]. In these cases, however, the fermion localization does not occur in the present one wall model. See Sec.6. )

We now understand that the periodic eigen functions $\left\{\mathrm{e}^{i k \mu y}\right\}$ in the KK case $((4)$ and $(17))$ correspond to $\left\{\xi_{k}(y), \eta_{k}(y)\right\}$ specified by (41). Only for the thin wall limit, they are explicitly solved by Bessel functions. Although we do not explicitly require the compactness of the extra space, the eigen values are $S^{1}$-like. This is due to the requirement of the geometry $\operatorname{AdS}_{5}\left(\approx \mathrm{S}^{1} \times \mathrm{R}^{4}\right)$ in the asymptotic region.

The importance of the 5D mass "function" $\hat{m}(y)$ is now clear. In the next section, we explain its origin in the bulk field theory. Nature requires the Yukawa interaction between the 5D fermion and the 5D Higgs[20].

\section{Bulk Higgs Mechanism}

One of the most important characters of the brane world model is the massless chiral fermion localization. It is phenomenologically attractive because 
the smallness of the quark and lepton masses could be naturally explained. Theoretically it is also necessary as the dimensional reduction mechanism. The feature comes from the $Z_{2}(y \leftrightarrow-y)$ properties of the system. The most natural way to introduce the properties is to use the Higgs mechanism in the bulk world. ${ }^{9}$

Let us examine the case the fermion system has the Yukawa coupling with the bulk Higgs field.

$$
\sqrt{-\hat{g}} \mathcal{L}=\sqrt{-\hat{g}}\left(\mathcal{L}^{\text {Dirac }}+\mathcal{L}^{Y}\right) \quad, \quad \mathcal{L}^{Y}=i g_{Y} \overline{\hat{\psi}} \hat{\psi} \Phi
$$

where the Higgs field $\Phi$ is the $5 \mathrm{D}$ (bulk) scalar field and $g_{Y}$ is the Yukawa coupling. We assume that the Higgs field, besides the "scale factor" field $\sigma(y)$, is some background given by the (classical) field equation of the 5D gravity-Higgs system.

$$
\mathcal{L}^{\text {grav }}=\frac{-1}{2 G_{5}} \hat{R} \quad, \quad \mathcal{L}^{S}=-\frac{1}{2} \nabla_{m} \Phi \nabla^{m} \Phi-V(\Phi), \quad,
$$

where $V(\Phi)$ is the ordinary Higgs potential. In ref.[14, 21], it is shown that the above gravity-Higgs system has a stable kink (domain wall) solution for the case $\Phi=\Phi(y)$. In the IR asymptotic region from the wall, $\sigma^{\prime}(y)$ and $\Phi(y)$ behave as

$$
\sigma^{\prime}(y)=\left\{\begin{array}{ll}
+\omega, & k y \rightarrow+\infty \\
-\omega, & k y \rightarrow-\infty
\end{array} \quad, \quad \Phi(y)=\left\{\begin{array}{ll}
+v_{0}, & k y \rightarrow+\infty \\
-v_{0}, & k y \rightarrow-\infty
\end{array},\right.\right.
$$

where $k$ (the inverse of thickness), $\omega$ (brane tension) and $v_{0}(5 \mathrm{D}$ Higgs vacuum expectation value) are some positive constants expressed by a free parameter, the vacuum parameters and the 5D gravitational constant. Near the origin of the extra axis $(k|y| \ll 1)$, they behave as

$$
\sigma^{\prime}(y)=\omega \tanh (k y) \quad, \quad \Phi(y)=v_{0} \tanh (k y) \quad .
$$

The dimensional reduction to $4 \mathrm{D}$ is performed by taking the the thin wall limit $k \rightarrow \infty$, which is precisely defined as

$$
k \gg \frac{1}{r_{c}} \text {. }
$$

\footnotetext{
${ }^{9}$ In the case of the flat space-time, Rubakov and Shaposhnikov[19] proposed a domain wall model caused by the bulk Higgs potential.
} 
where $r_{c}$ is the infrared cutoff of the extra axis $\left(-r_{c}<y<r_{c}\right)$. (See ref.[14].) In this limit, above quantities behave as $\sigma^{\prime}(y) \rightarrow \omega \theta(y), \Phi(y) \rightarrow v_{0} \theta(y)$. All dimensional parameters are a) $G_{5}{ }^{-1 / 3}$ : 5D Planck mass; b) $|\hat{m}|=g_{Y} v_{0}: 5 \mathrm{D}$ fermion mass; c) $k^{-1}$ : thickness of the domain; d) $r_{c}$ : Infrared regularization of the extra axis. Among them there exists a theoretical restriction from the requirement: 5D classical treatment works well.

$$
\frac{1}{\sqrt[3]{G_{5}}} \gg k
$$

The 5D Dirac equation of (49) is given by (cf. eq.(35)),

$$
i \mathrm{e}^{\sigma}\left\{\gamma^{a} \partial_{a}-2 \mathrm{e}^{-\sigma}\left(\sigma^{\prime}-\frac{1}{2} \partial_{y}\right) \gamma^{5}+g_{Y} \mathrm{e}^{-\sigma} \Phi\right\} \hat{\psi}=0
$$

This is just the lagragian of (35) with $\hat{m}(y)=g_{Y} \Phi(y)$. Let us examine a solution of the right chirality zero mode.

$$
\hat{\psi}(x, y)=\psi_{R}^{0}(x) \eta(y) \quad, \quad \gamma^{5} \psi_{R}^{0}=+\psi_{R}^{0} \quad, \quad \gamma^{a} \partial_{a} \psi_{R}^{0}=0 \quad .
$$

The equation (55) reduces to

$$
\partial_{y} \eta=\left(2 \sigma^{\prime}+g_{Y} \Phi(y)\right) \eta
$$

In the IR asymptotic region $(k|y| \gg 1)$, the solution behaves as

$$
\eta(y)=\text { const } \times \mathrm{e}^{-\left(g_{Y} v_{0}-2 \omega\right)|y|},
$$

which shows the exponentially damping for the large yukawa coupling $g_{Y}>$ $2 \omega / v_{0}[20]$. This is called massless chiral fermion localization. Near the origin of the extra axis $(k|y| \ll 1), \eta(y)$ behaves as

$$
\eta(y)=\text { const } \times \mathrm{e}^{-\frac{k}{2}\left(g_{Y} v_{0}-2 \omega\right) y^{2}},
$$

which shows the Gaussian damping. The behavior is shown in Fig.2. It shows the regular property of the solution. For the left chirality zero-mode, we can show the same behavior using the anti-kink solution instead of the kink solution (51). 


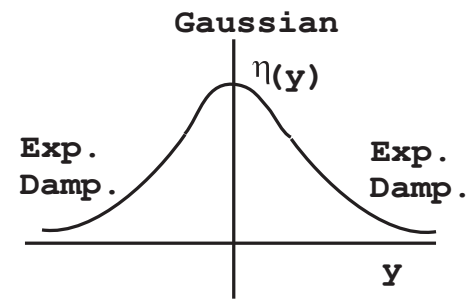

Fig.2 Behavior of the fermion along the extra axis.

\section{Five Dimensional QED and Bulk Quantum Effect}

Let us examine the 5D QED, $\mathcal{L}^{Q E D}=-e \overline{\hat{\psi}} \gamma^{\mu} \hat{e}_{\mu}^{m} \hat{\psi} A_{m}$, with the Yukawa interaction in RS geometry. ${ }^{10}$

$$
\begin{array}{r}
\sqrt{-\hat{g}}\left(\mathcal{L}^{\text {Dirac }}+\mathcal{L}^{Q E D}+\mathcal{L}^{Y}\right) \\
=\sqrt{-\hat{g}}\left[i \overline{\hat{\psi}}\left\{\gamma^{\mu} \hat{e}_{\mu}^{m}\left(\partial_{m}+i e A_{m}\right)+\frac{1}{8}\left(\hat{\omega}^{\sigma}\right)_{\mu \nu} \gamma_{\sigma}\left[\gamma^{\mu}, \gamma^{\nu}\right]\right\} \hat{\psi}+i g_{Y} \overline{\hat{\psi}} \hat{\psi} \Phi\right] .
\end{array}
$$

The kinetic (propagator) part for the electromagnetic, gravitational and Higgs fields is provided by

$$
\sqrt{-\hat{g}}\left(\mathcal{L}^{E M}+\mathcal{L}^{g r a v}+\mathcal{L}^{S}\right) \quad, \quad \mathcal{L}^{E M}=-\frac{1}{4} \hat{g}^{m n} \hat{g}^{k l} F_{m k} F_{n l}
$$

where $\mathcal{L}^{\text {grav }}, \mathcal{L}^{S}$ are given in (50). We assume, as in Sect.5 and $6, \hat{g}^{m n}$ and $\Phi$ are the brane background fields obtained as the stable solution of the system $\mathcal{L}^{\text {grav }}+\mathcal{L}^{S}$

We have introduced the Yukawa coupling in order to localize the fermion on the wall. This model, however, is still unsatisfactory in that the vector (gauge) field is not localized[20]. One resolution is to take 6D model[22]. Here we are content only with the fermion part and do not pursue this problem.

${ }^{10}$ The $\mathrm{U}(1)$ gauge space is here an internal space: $A_{m} \rightarrow A_{m}+\partial_{m} \Lambda(X), \hat{\psi} \rightarrow$ $\exp (-i e \Lambda(X)) \hat{\psi}$. This should be compared with the case of KK where the $\mathrm{U}(1)$ gauge space is identified with the extra $(y-)$ space. 


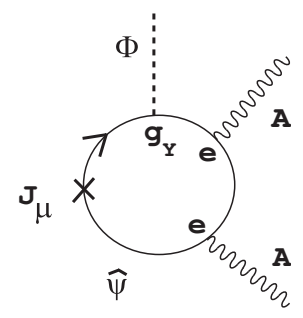

Fig.3 A bulk quantum-loop diagram. The diagram induces the effective action $S_{\text {eff }}^{(1)}$.

Let us examine the bulk quantum effect. It induces the 5D effective action $S_{\text {eff }}$ which reduces to a $4 \mathrm{D}$ action in the thin wall limit. From the diagram of Fig.3, we expect

$$
\frac{\delta S_{e f f}^{(1)}}{\delta A^{\mu}(X)} \equiv<J_{\mu}>\sim e^{2} g_{Y} \epsilon_{\mu \nu \lambda \sigma \tau} \Phi F^{\nu \lambda} F^{\sigma \tau}
$$

Then the effective action is integrated as

$$
S_{e f f}^{(1)} \sim e^{2} g_{Y} \int d^{5} X \epsilon_{\mu \nu \lambda \sigma \tau} \Phi A^{\mu} F^{\nu \lambda} F^{\sigma \tau}
$$

In the thin wall limit we may approximate as $\Phi=\Phi(y) \sim v_{0} \epsilon(y)$ where $\epsilon(y)$ is the step function. (See the description below (52).) Under the $\mathrm{U}(1)$ gauge transformation $\delta A^{\mu}=\partial^{\mu} \Lambda, S_{e f f}^{(1)}$ changes as

$$
\begin{array}{r}
\delta_{\Lambda} S_{e f f}^{(1)} \sim e^{2} g_{Y} v_{0} \int d^{5} X \epsilon_{\mu \nu \lambda \sigma \tau} \epsilon(y) \partial^{\mu} \Lambda F^{\nu \lambda} F^{\sigma \tau} \\
=e^{2} g_{Y} v_{0} \int d^{5} X\left\{\partial^{\mu}\left(\epsilon_{\mu \nu \lambda \sigma \tau} \epsilon(y) \Lambda F^{\nu \lambda} F^{\sigma \tau}\right)-\epsilon_{5 \nu \lambda \sigma \tau} \delta(y) \Lambda F^{\nu \lambda} F^{\sigma \tau}\right\} \\
=-e^{2} g_{Y} v_{0} \int d^{4} x \Lambda(x) F^{\alpha \beta} \tilde{F}_{\alpha \beta}
\end{array}
$$

where $\tilde{F}_{\alpha \beta} \equiv \epsilon_{\alpha \beta \gamma \delta} F^{\gamma \delta}$. In the above we assume that the boundary term vanishes. Callan and Harvey interpreted this result as the "anomaly flow" between the boundary (our 4D world) and the bulk[23]. Through the analysis of the induced action in the bulk, we can see the dual aspect of the 4D QED.

Another interesting bulk quantum effect is given by Fig.4. The induced 


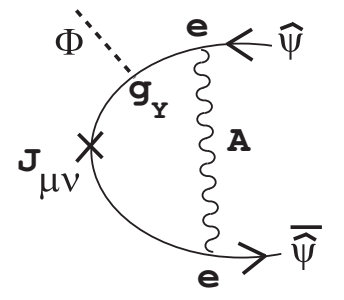

Fig.4 A bulk quantum-loop diagram. The diagram induces the effective action $S_{\text {eff }}^{(2)}$.

effective action $S_{\text {eff }}^{(2)}$ is expected to satisfy

$$
\frac{\delta S_{e f f}^{(2)}}{\delta F^{\mu \nu}} \equiv<J_{\mu \nu}>\sim e^{2} g_{Y} \epsilon_{\mu \nu \lambda \sigma \tau} \partial^{\lambda} \Phi \overline{\hat{\psi}} \Sigma^{\sigma \tau} \hat{\psi}
$$

Then $S_{e f f}^{(2)}$ is obtained as, in the thin wall limit,

$$
\begin{aligned}
S_{e f f}^{(2)} & \sim e^{2} g_{Y} \epsilon_{\mu \nu \lambda \sigma \tau} \int d^{5} X \partial^{\lambda} \Phi F^{\mu \nu} \overline{\hat{\psi}} \Sigma^{\sigma \tau} \hat{\psi} \\
=e^{2} g_{Y} v_{0} \epsilon_{\alpha \beta \gamma \delta} \int d^{4} x F^{\alpha \beta} \bar{\psi} \sigma^{\gamma \delta} \psi & =-i e^{2} g_{Y} v_{0} \int d^{4} x F^{\alpha \beta} \bar{\psi} \gamma^{5} \sigma_{\alpha \beta} \psi
\end{aligned}
$$

This term is the EDM term of (22). It violates the CP-symmetry. The coupling depends on the vacuum expectation value of $\Phi, v_{0}=\langle\Phi>$ which are, at present, not known. In order to estimate the magnitude of the coupling, it is necessary to apply this model to the quark-lepton (electro-weak) theory and fix the value. We expect the magnitude could be sufficiently large so that the result can be tested by present or near-future experiments.

The appearance of the EDM term corresponds to the CP-extremelyviolated case (ii) $|\hat{m}| \gg \mu$ of Sec.3. We compare the parameter relation with the thin wall relation (53) $k \gg 1 / r_{c}$. Because the parameter $\mu$ in KK case corresponds to $1 / r_{c}$ in RS case, we notice the $5 \mathrm{D}$ fermion mass in $\mathrm{KK}$ case $(|\hat{m}|)$ corresponds to the inverse of the thickness in RS case $(k)$.

The more fascinating view on the correspondence is that the $R S$ approach and the KK one are "dual" each other. We compare the above thin wall limit with the small radius limit (i) $|\hat{m}| \ll \mu$ of Sec.3. The thin wall limit, which is regarded as the dimensional reduction, can be consistently taken in RS 
model and EDM term naturally appears there. CP is not preserved. The theoretical treatment is justified as far as the relations (53) and (54) are satisfied: $1 / \sqrt[3]{G_{5}} \gg k \gg 1 / r_{c}$. While, in the KK case, the dimensional reduction takes place in the case (i) of Sec.3, which can be controlled theoretically as far as the relation $(26) 1 / \sqrt[3]{G_{5}}=\sqrt[3]{100} \mu \geq \mu \gg|\hat{m}|$ is satisfied. MDM term naturally appears and $\mathrm{CP}$ is preserved here.

In the effective action evaluation, the bulk Higgs field $\Phi(y)$ plays an important role. It serves as a bridge between the bulk world and the $4 \mathrm{D}$ world.

\section{Discussion and Conclusion}

Recently the higher dimensional (5D,6D) theories (with boundary terms) have been vigorously investigated in order to make them some consistent quantum field theories. In the past they were not so seriously examined because the renormalizability condition strictly excludes the higher dimensional theories. (One of few exceptions is the success of the vacuum energy calculation of the 5D Kaluza-Klein theory [24].) This past attitude was changed by the progress of the string and D-brane physics. Especially the AdS/CFT correspondence[25] gave us the image of the $4 \mathrm{D}$ world as a boundary of a higher dimensional space-time. The most crucial point is to find how the ordinary 4D quantum field theory is generalized to the extra-space direction. The treatment of the extra axis(es) becomes the central concern. In this point, some interesting proposals begin to be given by Goldberger-Wise[26] and by Randall-Schwartz[27, 28]. Both regard the extra space as the scale parameter space of the renormalization group. Renormalization flow moves in the extra space. New regularizations[29] are also proposed in order to regularize the summation over the all KK modes in the extra space direction. We comment that, in connection with the chiral determinant calculation based on the domain wall configuration in the $5 \mathrm{D}$ space-time, a new regularization is proposed[30, 31], where the extra space is regarded as the temperature (proper time) space. The present approach to this bulk quantum theory is different from the above ones. We take the induced effective action method. It has been used in relation to the chiral and Weyl anomalies. Famous successful ones are 2D WZNW model derived from the 2D QCD [32] and 2D induced quantum gravity[33]. We have examined mainly the thin wall limit. In order to examine the configuration off the limit, we must take into account 
the new proposals mentioned above. We should pursue this line of research.

We have comparatively examined the KK model and the RS model. Both have attractive features as the higher dimensional unification models. Using the Cartan formalism, fermions are properly treated. The KK expansion and the RS expansion are compared. The periodic functions appear in the former case, while the Bessel functions characteristically appear in the latter case. The dual property is controlled by two scale parameters, $\hat{m}$ and $\mu$ in the KK case whereas $k$ and $r_{c}$ in the RS case. In particular we stress that, as was pointed out by Thirring for the KK case, the CP-violation term naturally appears also in the RS model.

Finally we list the correspondence in Table 1. 


\begin{tabular}{|c|c|c|}
\hline & Kaluza-Klein & Randall-Sundrum \\
\hline electric charge & $e=f \mu, f$ :free para. & $e:$ free para. \\
\hline $\mathrm{U}(1)$ sym. & $\begin{array}{c}y \rightarrow y+\Lambda(x), \text { transl. in }\{\mathrm{y}\} \\
A_{a}(x) \rightarrow A_{a}(x)+\frac{1}{f} \partial_{a} \Lambda\end{array}$ & $\begin{array}{c}A_{m} \rightarrow A_{m}+\partial_{m} \Lambda, \text { internal sym. } \\
\quad\left(X^{m}\right)=\left(x^{a}, y\right): \text { fixed }\end{array}$ \\
\hline asym. geometry & $S^{1} \times \mathcal{M}_{4}$ & $\operatorname{AdS}_{5}\left(\omega, \tilde{m}=g_{Y} v_{0}\right)$ \\
\hline vacuum & $\begin{array}{c}\text { a massive KK mode for } \\
\text { charged fermion } \psi \text {, } \\
\text { 0-th KK modes for } \\
g_{a b}, A_{a}, \sigma \text { and } \\
\text { neutral fermion }\end{array}$ & $\begin{array}{l}\text { 5D bulk Higgs vacuum } \\
<\Phi>= \pm v_{0}, y \rightarrow \pm \infty \\
\text { kink sol., } Z_{2} \text {-symmetry }\end{array}$ \\
\hline 4D fermion mass & $\sqrt{\hat{m}^{2}+\mu^{2}}$ & $g_{Y} v_{0} \times$ overlap-int \\
\hline physical scale & $\hat{m}: 5 \mathrm{D}$ fermion mass & $k:\left(\right.$ thickness $^{-1}$ \\
\hline global size & $\begin{array}{c}\mu^{-1} \text { :radius of extra } S^{1} \\
y \rightarrow y+2 \pi \mu^{-1} \text {, periodic }\end{array}$ & $\begin{array}{l}r_{c}: \text { IR cutoff } \\
-r_{c} \leq y \leq r_{c}\end{array}$ \\
\hline $\begin{array}{l}\text { dimensional } \\
\text { reduction cond. }\end{array}$ & $\begin{array}{c}\mu \gg \hat{m} \\
\text { small radius limit }\end{array}$ & $\begin{array}{c}\qquad k \gg 1 / r_{c} \\
\text { thin-wall limit }\end{array}$ \\
\hline $\begin{array}{l}\text { 5D classical } \\
\text { condition }\end{array}$ & $1 / \sqrt[3]{G_{5}} \sim \sqrt[3]{100} \mu \gg \hat{m}$ & $1 / \sqrt[3]{G_{5}} \gg k$ \\
\hline $\begin{array}{l}\text { mode functions } \\
\text { in extra space }\end{array}$ & $\begin{array}{l}\mathrm{e}^{i k \mu y}, k \in \mathbf{Z} \\
\text { periodic func. }\end{array}$ & $\begin{array}{c}J_{\nu}\left(m_{k} z\right), N_{\nu}\left(m_{k} z\right), \nu=\left|\frac{1}{2}-\tilde{m} / \omega\right| \\
z \omega=\mathrm{e}^{\omega y}, k \in \mathbf{Z}, \text { Bessel func. }\end{array}$ \\
\hline CP property & $\begin{array}{l}\text { MDM in small radius } \\
\text { limit, CP-preserved }\end{array}$ & $\begin{array}{l}\text { EDM in thin wall } \\
\text { limit, CP-violated }\end{array}$ \\
\hline
\end{tabular}

Table 1 Comparison of KK model and RS model.

\section{Acknowledgment}

The author thanks K. Akama for some comments and precious information about the brane world. He also thanks S.D. Odintsov for bringing the references $[27,28]$ to his attention. Parts of the present results were presented at Chubu Summer School (Syuzenji, Japan, 2001.8.30-9.2), the 5th KEK Topical Conference -Frontiers in Flavor Physics- (Tsukuba, Japan, 2001.11.2022), YITP Workshop on "Fundamental Problems and Applications in Quantum Field Theory" (Kyoto, Japan, 2001.12.19-21), the annual meeting of the Physical Society of Japan (Ritsumeikan Univ.,Kusatsu, Japan, 2002.3.24-27) 
and Summer Institute 2002 (Fuji-Yoshida, Japan, 2002.8.13-20). Discussions with the audience are appreciated. In particular the author thanks T. Inami, T. Kugo, M. Nakahara and N. Nakanishi.

\section{Appendix A 5D Dirac Matrix}

In order to clearly discuss (5D) Dirac equation, we must fix the representation of the gamma matrices. The signs and phases appearing in the text depend on the convention. We take the Majorana representation for the Dirac matrix.

$$
\begin{gathered}
\left\{\gamma_{\mu}, \gamma_{\nu}\right\}=2 \eta_{\mu \nu} \quad, \quad\left(\eta_{\mu \nu}\right)=\operatorname{diag}(-1,1,1,1,1) \\
\mu, \nu=0,1,2,3,5 \\
\gamma_{0}=-\gamma^{0}=\left(\begin{array}{cc}
0 & i \sigma_{2} \\
i \sigma_{2} & 0
\end{array}\right) \quad, \quad \gamma_{1}=\gamma^{1}=\left(\begin{array}{cc}
\sigma_{1} & 0 \\
0 & \sigma_{1}
\end{array}\right) \\
\gamma_{2}=\gamma^{2}=\left(\begin{array}{cc}
0 & -i \sigma_{2} \\
i \sigma_{2} & 0
\end{array}\right) \quad, \quad \gamma_{3}=\gamma^{3}=\left(\begin{array}{cc}
\sigma_{3} & 0 \\
0 & \sigma_{3}
\end{array}\right) \\
\gamma_{5}=\gamma^{5}=i \gamma_{0} \gamma_{1} \gamma_{2} \gamma_{3}=-i \gamma^{0} \gamma^{1} \gamma^{2} \gamma^{3}=\left(\begin{array}{cc}
\sigma_{2} & 0 \\
0 & -\sigma_{2}
\end{array}\right)
\end{gathered}
$$

where $\sigma_{i}(i=1,2,3)$ are the Pauli matrices: $\sigma_{1}=(0,1 / 1,0), \sigma_{2}=(0,-i / i, 0), \sigma_{3}=$ $(1,0 / 0,-1)$. The above gamma matrices have the following properties.

- $\gamma_{0}, \gamma_{1}, \gamma_{2}, \gamma_{3}$ are real matrices, while $\gamma_{5}$ is pure imaginary.

- $\gamma_{1}, \gamma_{2}, \gamma_{3}, \gamma_{5}$ are hermitian, while $\gamma_{0}$ is anti-hermitian. $\gamma_{0} \gamma_{\mu}^{\dagger} \gamma_{0}=\gamma_{\mu}$

- $\gamma_{1}, \gamma_{2}, \gamma_{3}$ are symmetric, while $\gamma_{0}, \gamma_{5}$ are anti-symmetric.

- $\gamma_{0}^{2}=-1, \gamma_{1}^{2}=\gamma_{2}^{2}=\gamma_{3}^{2}=\gamma_{5}^{2}=1$ 


\section{Appendix B Randall-Sundrum Geometry in the Cartan Formalism}

We take the more general metric than (30) which is useful when the coordinate $y$ taken in the text is transformed to another coordinates.

$$
\begin{array}{r}
d s^{2}=\mathrm{e}^{-2 \sigma(z)} \eta_{a b} d x^{a} d x^{b}+\frac{4}{F(z)^{2}} d z^{2}, \\
-\infty<x^{a}<+\infty,\left(\eta_{a b}\right)=\operatorname{diag}(-1,1,1,1)
\end{array}
$$

where $a, b=0,1,2,3$. The coordinate taken in the text is the case: $F(z)=2$. If we denote this coordinate as $y$ as in the text, its coordinate region is $-\infty<$ $y<+\infty$ which shows non-compactness of the extra space. We can transform to some compact coordinates by the following coordinate transformations ${ }^{11}$ : 1) $z=\frac{1}{2} \tanh y,-\frac{1}{2}<z<\frac{1}{2}, F(z)=\left(1-4 z^{2}\right)$; 2) $y=\tan (\pi z),-\frac{1}{2}<z<$ $\frac{1}{2}, F(z)=(2 / \pi) \cos ^{2}(\pi z)$. We now start with $F(z)$ unspecified. Then the 5D metric is given as

$$
\begin{array}{r}
d s^{2}=\hat{g}_{m n} d X^{m} d X^{n} \quad, \quad\left(X^{m}\right)=\left(x^{a}, z\right) \\
\left(\hat{g}_{m n}\right)=\left(\begin{array}{cc}
\eta_{a b} \mathrm{e}^{-2 \sigma} & 0 \\
0 & \frac{4}{F(z)^{2}}
\end{array}\right)
\end{array}
$$

where $m, n=0,1,2,3,5$. From these the basis of the cotangent manifold $\mathrm{T}_{p}^{*} \mathrm{M}, \theta^{\mu}$, is given as

$$
\begin{array}{r}
d s^{2}=\theta^{\mu} \theta^{\nu} \hat{\eta}_{\mu \nu}, \quad\left(\hat{\eta}_{\mu \nu}\right)=\operatorname{diag}(-1,1,1,1,1) \\
\theta^{\alpha}=\mathrm{e}^{-\sigma} \eta_{a}^{\alpha} d x^{a} \quad, \quad \theta^{\overline{5}}=\frac{2}{F(z)} d z
\end{array}
$$

where $\mu, \nu=0,1,2,3, \overline{5}$ and $\alpha=0,1,2,3$. From the above result we can read the fünf-bein $\mathrm{e}_{m}^{\mu}$.

$$
\left(\mathrm{e}_{m}^{\mu}\right)=\left(\begin{array}{cc}
\eta^{\alpha} \mathrm{e}^{-\sigma} & 0 \\
0 & \frac{2}{F(z)}
\end{array}\right) \quad, \quad\left(\mathrm{e}_{\mu}{ }^{m}\right)=\left(\begin{array}{cc}
\eta_{\alpha}{ }^{a} \mathrm{e}^{\sigma} & 0 \\
0 & \frac{1}{2} F(z)
\end{array}\right),
$$

\footnotetext{
${ }^{11}$ These coordinates are important to give some renormalization group interpretation to the RS model[34].
} 
where $\mathrm{e}_{\mu}^{m}$ is the inverse of $\mathrm{e}_{m}^{\mu}$. The basis of the tangent manifold $\mathrm{T}_{p} \mathrm{M}, \mathrm{e}_{\mu}$, is given by

$$
\begin{aligned}
& \mathrm{e}_{\mu} \equiv \mathrm{e}_{\mu}^{m} \frac{\partial}{\partial X^{m}} \\
& \mathrm{e}_{\alpha}=\mathrm{e}^{\sigma} \eta_{\alpha}{ }^{a} \frac{\partial}{\partial x^{a}} \quad, \quad \mathrm{e}_{\overline{5}}=\frac{1}{2} F(z) \frac{\partial}{\partial z} .
\end{aligned}
$$

$\left\{\mathrm{e}_{\mu}\right\}=\left\{\mathrm{e}_{\alpha}, \mathrm{e}_{\overline{5}}\right\}$ and $\left\{\theta^{\mu}\right\}=\left\{\theta^{\alpha}, \theta^{\overline{5}}\right\}$ constitute the non-coordinate basis, while $\left\{\mathrm{e}_{m} \equiv \frac{\partial}{\partial X^{m}}\right\}=\left\{\frac{\partial}{\partial x^{a}}, \frac{\partial}{\partial z}\right\}$ and $\left\{d X^{m}\right\}=\left\{d x^{a}, d z\right\}$ constitute the coordinate basis.

The Lie algebra between $\left\{\mathrm{e}_{\mu}\right\}$ is obtained as

$$
\left[\mathrm{e}_{\alpha}, \mathrm{e}_{\beta}\right]=\left[\mathrm{e}_{\overline{5}}, \mathrm{e}_{\overline{5}}\right]=0, \quad\left[\mathrm{e}_{\alpha}, \mathrm{e}_{\overline{5}}\right]=\frac{1}{2} F \sigma^{\prime} \mathrm{e}_{\alpha},
$$

where $" \prime "=\frac{d}{d z}$. The "structure constants", $c_{\mu \nu}{ }^{\lambda}$, are read as

$$
\begin{array}{r}
{\left[\mathrm{e}_{\mu}, \mathrm{e}_{\nu}\right]=c_{\mu \nu}{ }^{\lambda} \mathrm{e}_{\lambda},} \\
c_{\alpha \beta}^{\mu}=c_{\overline{5} \overline{5}}{ }^{\mu}=0, c_{\alpha \overline{5}}{ }^{\beta}=-c_{\overline{5} \alpha}{ }^{\beta}=\frac{1}{2} F \sigma^{\prime} \eta_{\alpha}{ }^{\beta}, c_{\alpha \overline{5}}{ }^{\overline{5}}=-c_{\overline{5} \alpha}{ }^{\overline{5}}=0
\end{array} .
$$

The first Cartan's structure equation, for the torsionless case, is given by

$$
d \theta^{\mu}+\omega_{\nu}^{\mu} \wedge \theta^{\nu}=T^{\mu}=0
$$

where $\omega^{\mu}{ }_{\nu}$ is the connection one-form. Using the obtained expression of $\theta^{\mu}$, the above equation serves for fixing the connection.

$$
\begin{aligned}
& \text { (i) } \underline{\mu=\alpha, \theta^{\alpha}=\mathrm{e}^{-\sigma} d x^{\alpha}} \quad: \quad \omega_{5}^{\alpha}=-\omega_{\overline{5}}^{\alpha}=-\frac{\sigma^{\prime}}{2} F \theta^{\overline{5}} \quad, \quad \omega_{\beta}^{\alpha}=0 \text {, } \\
& \text { (ii) } \mu=\overline{5}, \theta^{\overline{5}}=\frac{2}{F(z)} d z \quad: \quad \omega_{\alpha}^{\overline{5}}=\frac{\sigma^{\prime}}{2} F \theta_{\alpha} \quad, \quad \theta_{\alpha}=\eta_{\alpha \beta} \theta^{\beta} \\
& \text { (iii) } \underline{\omega_{\mu \nu}=-\omega_{\nu \mu}, \omega_{\nu}^{\mu}=-\omega_{\nu}^{\mu}}: \omega_{\overline{5}}^{\overline{5}_{\bar{T}}}=0 \text {. }
\end{aligned}
$$

Now we can calculate the curvature using the second Cartan's structure equation.

$$
d \omega^{\mu}{ }_{\nu}+\omega_{\lambda}^{\mu} \wedge \omega_{\nu}^{\lambda}=R_{\nu}^{\mu}=\frac{1}{2} R_{\nu \lambda \sigma}^{\mu} \theta^{\lambda} \wedge \theta^{\sigma}
$$


where $R^{\mu}{ }_{\nu}$ is the curvature 2 -form. They are given as

$$
\begin{array}{r}
(i) \underline{\mu=\alpha, \nu=\beta} \\
R_{\beta \overline{5} \overline{5}}^{\alpha}=R_{\beta \gamma \overline{5}}^{\alpha}=-R_{\beta \overline{5} \gamma}^{\alpha}=0, R_{\beta \gamma \delta}^{\alpha}=-\frac{\sigma^{\prime 2}}{4} F^{2}\left(\eta_{\gamma}^{\alpha} \eta_{\beta \delta}-\eta_{\delta}^{\alpha} \eta_{\beta \gamma}\right), \\
(i i) \underline{\mu=\overline{5}, \nu=\overline{5}} \quad: \quad R_{\overline{5} \mu \nu}^{\overline{5}}=0, \\
(i i i) \underline{\mu=\alpha, \nu=\overline{5}} \quad: \quad R_{5 \overline{5} \beta}^{\alpha}=-R_{\overline{5} \beta \overline{5}}^{\alpha}=-\frac{1}{4}\left(\sigma^{\prime} F \mathrm{e}^{-\sigma}\right)^{\prime} F \mathrm{e}^{\sigma} \eta_{\beta}^{\alpha}, \\
(i v) \underline{\mu=\overline{5}, \nu=\alpha} \quad: \quad R_{\alpha \overline{5} \beta}^{\bar{\mu}}=-R_{\alpha \beta \overline{5}}^{\overline{5}}=\frac{1}{4}\left(\sigma^{\prime} F \mathrm{e}^{-\sigma}\right)^{\prime} F \mathrm{e}^{\sigma} \eta_{\alpha \beta} \quad .
\end{array}
$$

The coordinate components are obtained by multiplying the fünf-beins.

$$
\begin{array}{r}
R_{n k l}^{m}=\mathrm{e}_{\mu}{ }^{m} \mathrm{e}_{n}^{\nu} \mathrm{e}_{k}^{\lambda} \mathrm{e}_{l}^{\sigma} R_{\nu \lambda \sigma}^{\mu} \quad, \quad R_{m n}=R_{m n k}^{k}, \\
R_{55}=-\frac{4}{F}\left(\sigma^{\prime \prime} F+\sigma^{\prime} F^{\prime}-\sigma^{\prime 2} F\right), \\
R_{a b}=-\frac{1}{4} F \mathrm{e}^{-2 \sigma}\left(\sigma^{\prime \prime} F+\sigma^{\prime} F^{\prime}-4 \sigma^{\prime 2} F\right) \eta_{a b} \quad, \quad R_{5 a}=R_{a 5}=0 .
\end{array}
$$

From the connection 1-form $\omega_{\nu}^{\mu}$, we can define the connection coefficients as follows.

$$
\begin{aligned}
& \omega^{\mu}{ }_{\nu}=\Gamma_{\lambda \nu}^{\mu} \theta^{\lambda} \\
& \text { (i) } \underline{\omega_{\overline{5}}^{\alpha}=-\frac{\sigma^{\prime}}{2} F \theta^{\alpha}} \quad: \quad \Gamma_{\beta \overline{5}}^{\alpha}=-\frac{\sigma^{\prime}}{2} F \eta_{\beta}^{\alpha} \quad, \quad \Gamma_{\overline{5} \overline{5}}^{\alpha}=0 . \\
& \text { (ii) } \left.\underline{\omega_{\overline{5}}^{\overline{5}}=\omega_{\beta}^{\alpha}=0} \quad: \quad \Gamma_{\mu \overline{5}}^{\overline{5}}=\Gamma_{\mu \beta}^{\alpha}=0 \quad \text {. (especially } \Gamma_{\overline{5}_{\beta}}^{\alpha}=0 .\right) \\
& \text { (iii) } \underline{\omega_{\alpha}^{\overline{5}}=\frac{\sigma^{\prime}}{2} F \theta_{\alpha}} \quad: \quad \Gamma_{\beta \alpha}^{\overline{5}}=\frac{\sigma^{\prime}}{2} F \eta_{\beta \alpha} \quad, \quad \Gamma_{\overline{5}_{\alpha}}^{\overline{5}}=0 .
\end{aligned}
$$

Note that generally $\Gamma_{\lambda \nu}^{\mu} \neq \Gamma_{\nu \lambda}^{\mu}$. Indeed the above result satisfies the torsionless condition, for example, $T_{5 \beta}^{\alpha} \equiv \Gamma_{5 \beta}^{\alpha}-\Gamma_{\beta \overline{5}}^{\alpha}-c_{\overline{5} \beta}^{\alpha}=0$.

The connection coefficient $\Gamma_{\mu \nu}^{\lambda}$ are related with the same one with respect to $\left\{e_{m}\right\}$ as

$$
\begin{gathered}
\Gamma^{\lambda}{ }_{\mu \nu}=\mathrm{e}_{n}^{\lambda} \mathrm{e}_{\mu}{ }^{m}\left(\partial_{m} \mathrm{e}_{\nu}{ }^{n}+\mathrm{e}_{\nu}{ }^{k} \Gamma^{n}{ }_{m k}\right), \\
\Gamma_{m n}^{k}=\mathrm{e}_{\lambda}^{k} \mathrm{e}_{m}^{\mu}{ }_{m} \mathrm{e}_{n}^{\nu} \Gamma^{\lambda}{ }_{\mu \nu}-\partial_{m} \mathrm{e}_{\mu}{ }^{k} \mathrm{e}_{n}^{\mu} .
\end{gathered} .
$$


This formula gives

$$
\Gamma_{55}^{5}=-\frac{F^{\prime}}{F} \quad, \quad \Gamma_{a b}^{5}=\frac{\sigma^{\prime}}{4} F^{2} \mathrm{e}^{-2 \sigma} \eta_{a b} \quad, \quad \Gamma_{5 b}^{a}=\Gamma_{b 5}^{a}=-\sigma^{\prime} \eta_{b}^{a} .
$$

The connection coefficient with the "mixed type" $\Gamma_{m \nu}^{\mu}$, is defined by

$$
\begin{gathered}
\Gamma_{m \nu}^{\mu} \equiv \Gamma_{\lambda \nu}^{\mu} \mathrm{e}_{m}^{\lambda} \\
\omega_{\nu}^{\mu}=\Gamma_{\lambda \nu}^{\mu} \theta^{\lambda}=\Gamma_{\lambda \nu}^{\mu} \mathrm{e}_{m}^{\lambda} d X^{m}=\Gamma_{m \nu}^{\mu} d X^{m}
\end{gathered} .
$$

This formula gives

$$
\begin{aligned}
& \text { (i) } \omega_{\overline{5}}^{\overline{5}}=0 \quad, \quad \omega_{\beta}^{\alpha}=0 \quad: \quad \Gamma_{m \overline{5}}^{\overline{5}}=\Gamma_{m \beta}^{\alpha}=0, \\
& \text { (ii) } \omega_{\overline{5}}^{\alpha}=-\frac{\sigma^{\prime}}{2} F \mathrm{e}^{-\sigma} d x^{\alpha} \quad: \quad \Gamma_{5 \overline{5}}^{\alpha}=0 \quad, \quad \Gamma_{a \overline{5}}^{\alpha}=-\frac{\sigma^{\prime}}{2} F \mathrm{e}^{-\sigma} \eta_{a}^{\alpha} \text {, } \\
& \text { (iii) } \omega_{\alpha}^{\overline{5}}=\frac{\sigma^{\prime}}{2} F \mathrm{e}^{-\sigma} d x^{\alpha} \quad: \quad \Gamma_{5 \alpha}^{\overline{5}}=0 \quad, \quad \Gamma_{a \alpha}^{\overline{5}}=\frac{\sigma^{\prime}}{2} F \mathrm{e}^{-\sigma} \eta_{a \alpha} .
\end{aligned}
$$

\section{References}

[1] S. Ichinose, Proc. Fifth KEK Topical Conference (2001.11.19-22, KEK,Tsukuba,Japan), ed. S. Hashimoto and T.K. Komatsubara, to be published in Nucl.Phys.B(Proc. Suppl.), "CP-Violation in Kaluza-Klein and Randall-Sundrum Theories"

[2] Th. Kaluza, Sitzungsberichte der K.Preussischen Akademite der Wissenschaften zu Berlin. p966 (1921).

[3] O. Klein, Z. Physik 37895 (1926).

[4] L.Randall and R.Sundrum, Phys.Rev.Lett.83(1999)3370,hep-ph/9905221

[5] L.Randall and R.Sundrum, Phys.Rev.Lett.83(1999)4690,hep-th/9906064 
[6] K. Akama, T. Hattori and K. Katsuura, Phys.Rev.Lett.88(2002)201601, hep-ph/0111238

[7] W. Thirring, Acta Physica Austriaca, Suppl.IX, 256-271(1972), Springer-Verlag, "Fivedimensional Theories and CP-Violation"

[8] Y. Sakamura, hep-th/0011098, "Our wall as the origin of cp violation"

[9] D. Dooling, D.A. Easson and K. Kang, hep-ph/0202206, "Geometric Origin of CP Violation in an Extra-Dimensional Brane World"

[10] É. Cartan, "Leçons sur la Géométrie des Espaces de Riemann", Gauthier-Villars, Paris, France, 1928, 1946

[11] C.W. Misner, K.S. Thorne and J.A. Wheeler, "GRAVITATION", W.H. Freeman and Company, New York, USA, 1973, §14.5.

[12] R.M. Wald, "General Relativity", The Univ. of Chicago Press, Chicago and London, 1984, §3.4b

[13] M. Nakahara, "Geometry Topology and Physics", Adam Hilger, Bristol, England, 1990.

[14] S.Ichinose,Class.Quant.Grav.18(2001)421,hep-th/0003275

[15] P.G.Harris et al, Phys.Rev.Lett.82(1999)904

[16] S.Chang, J.Hisano, H.Nakano, N.Okada and M.Yamaguchi, Phys.Rev.D62(2000)084025, hep-ph/9912498,

[17] D.B.Kaplan and M.Schmaltz, Chinese Jour.Phys.38(2000)543 [Proc. of the Workshop on Chiral Gauge Theories, "Chiral '99", Sep.1318,1999, Taipei,Taiwan]

[18] Y.Grossman and M.Neubert, Phys.Lett.B474(2000)361, hep-ph/9912408

[19] V.A. Rubakov and M.E. Shaposhnikov, Phys.Lett.B125(1983)136

[20] B.Bajc and G.Gabadadze, Phys.Lett.B474(2000)282

[21] S.Ichinose,Class.Quant.Grav.18(2001)5239,hep-th/0107254 
[22] I.Oda, Phys.Lett.B496(2000)113, hep-th/0006203

[23] C.G.Callan and J.A.Harvey,Nucl.Phys.B250(1985)427

[24] T. Appelquist and A. Chodos, Phys.Rev.D28(1983)772; Phys.Rev.Lett.50(1983)141

[25] J.M.Maldacena, Adv.Theor.Math.Phys.2(1998)231;

Int.J.Theor.Phys.38(1999) 1113

[26] W.D. Goldberger and M.B. Wise, Phys.Rev.D65(2002)025011, hepth/0104170

[27] L. Randall and M.D. Schwartz, Phys.Rev.Lett.88(2002)081801, hepth/0108115,

[28] L. Randall and M.D. Schwartz, JHEP 0111:003,2001, hep-th/0108114

[29] S.G. Nibbelink, Nucl.Phys.B619(2001)373, hep-th/0108185; hepth/0111288, "Kaluza-Klein towers on orbifolds: divergences and anomalies"

[30] S. Ichinose, Nucl.Phys.B574(2000)719

[31] S. Ichinose, Prog.Theor.Phys.107(2002)1069

[32] A.M. Polyakov and P.B. Wiegmann, Phys.Lett.131B(1983)121

[33] A.M. Polyakov, Mod.Phys.Lett.A2(1987)893

[34] S.Ichinose, Phys.Rev.D65(2002)084038, hep-th/0008245, 